\title{
IL-37 Gene Modification Enhances the Protective Effects of Mesenchymal Stromal Cells on Intestinal Ischemia Reperfusion Injury
}

\section{Dejun Kong}

Department of General Surgery $₫ T i a n j i n$ Medical University General Hospital囚Tianjin General Surgery Institute

\section{Yonghao Hu}

Department of General Surgery®Tianjin Medical University General Hospital囚Tianjin General Surgery Institute

\section{Xiang Li}

Department of General Surgery®Tianjin Medical University General Hospital囚Tianjin General Surgery Institute

\section{Dingding Yu}

Department of General Surgery®Tianjin Medical University General Hospital囚Tianjin General Surgery Institute

\section{Hongyue Li}

Department of General Surgery $₫ T i a n j i n$ Medical University General Hospital囚Tianjin General Surgery Institute

\section{Yiming Zhao}

Department of General Surgery囚Tianjin Medical University General Hospital囚Tianjin General Surgery Institute

\section{Yafei Qin}

Department of General Surgery囚Tianjin Medical University General Hospital囚Tianjin General Surgery Institute

\section{Wang Jin}

Department of General Surgery $₫ T$ Tianjin Medical University General Hospital囚Tianjin General Surgery Institute

\section{Baoren Zhang}

Department of General Surgery囚Tianjin Medical University General Hospital囚Tianjin General Surgery Institute

\section{Bo Wang}

Department of Paediatric Surgery, Tianjin Medical University General Hospital Hongda Wang 
Department of General Surgery $₫$ Tianjin Medical University General Hospital\Tianjin General Surgery Institute

\section{Guangming Li}

Department of General Surgery $₫$ Tianjin Medical University General Hospital囚Tianjin General Surgery Institute

\section{Hao Wang ( $\nabla$ hwangca272@hotmail.com )}

Tianjin Medical University General Hospital, Tianjin General Surgery Institute https://orcid.org/00000001-9990-2714

\section{Research}

Keywords: IL 37, mesenchymal stromal cells, ischemia reperfusion injury, intestine

Posted Date: April 29th, 2020

DOI: https://doi.org/10.21203/rs.3.rs-24984/v1

License: (c) (1) This work is licensed under a Creative Commons Attribution 4.0 International License. Read Full License

Version of Record: A version of this preprint was published at Stem Cells International on August 8th, 2020. See the published version at https://doi.org/10.1155/2020/8883636. 


\section{IL-37 Gene Modification Enhances the Protective Effects of Mesenchymal Stromal Cells on Intestinal Ischemia Reperfusion Injury}

Dejun Kong ${ }^{1,2 \dagger}$, Yonghao $\mathrm{Hu}^{1,2 \dagger}$, Xiang $\mathrm{Li}^{1,2 \dagger}$, Dingding $\mathrm{Yu}^{1,2}$, Hongyue $\mathrm{Li}^{1,2}$, Yiming Zhao $^{1,2}$, Yafei Qin ${ }^{1,2}$, Wang Jin ${ }^{1,2}$, Baoren Zhang ${ }^{1,2}$, Bo Wang ${ }^{3}$, Hongda Wang ${ }^{1,2}$, Guangming $\mathrm{Li}^{1,2}$, Hao Wang ${ }^{1,2^{*}}$.

${ }^{\dagger}$ Dejun Kong, Yonghao Hu and Xiang Li are co-first authors on this paper.

${ }^{1}$ Department of General Surgery, Tianjin Medical University General Hospital, Tianjin, China;

${ }^{2}$ Tianjin General Surgery Institute, Tianjin, China;

${ }^{3}$ Department of Paediatric Surgery, Tianjin Medical University General Hospital, Tianjin, China.

\section{Email address for all authors}

Dejun Kong (DejunK@tmu.edu.cn)

Yonghao Hu (dryhhu@163.com)

Xiang Li (1x3325@126.com)

Dingding Yu (yuding4535@tmu.edu.cn)

Hongyue Li (xgllachel@163.com)

Yiming Zhao (yimingzh66@ hotmail.com)

Yafei Qin (qinyafei92@tmu.edu.cn)

Wang Jin (jingwang94@tmu.edu.cn) 
Baoren Zhang (zhangbaoren@tmu.edu.cn)

Bo Wang (wanbo412@163.com)

Hongda Wang (wanghd0326@163.com)

Guangming Li (liguang1118@163.com)

Hao Wang (hwangca272@hotmail.com or hwang1@tmu.edu.cn)

*Corresponding author: Dr. Hao Wang, Department of General Surgery, Tianjin Medical University General Hospital, 154 Anshan Road, Heping District, Tianjin 300052, China, Telephone: 01186-22-60362502, Email: hwangca272@ hotmail.com (preferred) or hwang1@tmu.edu.cn 


\section{Abstract:}

Background: Ischemia reperfusion injury (IRI) is the major cause of intestinal damage in clinic. Although either mesenchymal stromal cells (MSCs) or interleukin 37 (IL-37) showed some beneficial roles to ameliorate IRI, their effects are limited. In this study, the protective effects of IL-37 gene-modified MSCs (IL-37-MSCs) for better prevention of intestinal IRI are investigated.

Methods: Intestinal IRI model was established by occluding the superior mesenteric artery for $30 \mathrm{~min}$ and then reperfusing for 72 hours in rats. Forty adult male SD rats were randomly divided into sham control, IL-37-MSC-treated, MSC-treated, recombinant IL-37 (rIL-37)-treated and untreated groups. Intestinal damage was assessed by H\&E staining. The levels of gut barrier function factors (diamine oxidase and D-Lactate) and inflammation reactivity cytokine IL-1 $\beta$ were assayed by ELISA. The expressions of tissue damage-related NLRP3 inflammasome and relative proteins including clevead caspase-1, IL-1 $\beta$ and IL-18 were detected by western blot. As downstream of IL-1 $\beta$ and IL-18, the mRNA levels of proinflammatory mediators IL-6 and TNF- $\alpha$ were determined by qPCR. Data were analyzed by one-way analysis of variance among groups.

Results: IL-37-MSCs were able to migrate to the damaged tissue and significantly inhibit intestinal IRI. As compared with MSCs or rIL-37 monotherapy group, IL-37-MSC treatment not only improved gut barrier function but also decreased local and systemic inflammation reactivity cytokine IL-1 $\beta$ level in IRI rats. In addition, tissue damage-related NLRP3 and relative proteins (cleaved caspase-1, IL-1 $\beta$ and 
IL-18) were significantly decreased in IRI rats treated with IL-37-MSCs. Furthermore, IL-1 $\beta$ and IL-18 related proinflammatory mediators IL-6 and TNF- $\alpha$ mRNA expressions were markedly decreased following IL-37-MSC treatment.

Conclusion: The results suggest that IL-37 gene modification significantly enhance the protective effects of MSCs against intestinal IRI. In addition, NLRP3-related signaling pathways could be associated with IL-37-MSC mediated protection.

Key words: IL-37, mesenchymal stromal cells, ischemia reperfusion injury, intestine 


\section{Background:}

Intestinal ischemia-reperfusion injury (IRI), one of the major causes of clinical acute intestine necrosis, is a life-threatening disease with high mortality and disability [1]. Intestinal IRI is common in multiple diseases including vascular occlusion, haemorrhagic shock, trauma and small bowel transplantation etc [2, 3]. However, currently there is no effective treatment available besides surgical intervention, and thus a novel treatment is urgently needed [4].

The underlying mechanisms of intestinal IRI are complicated and diverse factors are involved in the process [1]. Briefly, intestinal IRI frequently derived from circulatory diseases in the gastrointestinal system involving the superior mesenteric artery (SMA), innate immunity, adaptive immunity and inflammation were essential in the development of IRI. At the early stage of ischemia, endothelial barrier is destroyed and then the vascular permeability will increase. Simultaneously, reactive oxygen species (ROS) can be excessively produced. After that, reperfusion upregulates the release of ROS, consequently disrupting normal ATP generation. Excessive ROS also cause severe oxidative stress, which can promote DNA damage, endothelial dysfunction and local inflammatory responses. On the basis of ischemia, reperfusion triggers the release of intracellular and extracellular damage-associated molecular pattern molecules (DAMPs), therefore, resulting in the accumulation of inflammatory cells such as monocytes, dendritic cells and so on [5].

The NOD-like receptor protein 3 (NLRP3) is a member of pattern recognition receptors and works as a key role in inflammatory response via forming an 
intracellular multi-protein complex known as NLRP3 inflammasome, which is the best characterized of all other inflammasomes [6]. Inflammasomes are important signal platforms in detecting sterile stressors and pathogenic microorganisms such as some DAMPs, which activate and release the highly pro-inflammatory cytokines interleukin-1 $\beta$ (IL-1 $\beta$ ) and interleukin-18 (IL-18). In this process, ROS as a kind of DAMPs was excessively produced during IRI and then promote NLRP3 inflammasome activation [7], subsequently promoting pro-caspase-1 into cleaved-caspase-1, the activation of caspase-1 can upregulate the production and secretion of the cytokines such as IL-1 $\beta$ and IL-18 by cleavage of pro-IL-1 $1 \beta$ and pro-IL-18. At the same time, activation of inflammasome-associated inflammatory caspase-1 drives cleavage of the pro-pyroptosis factor gasdermin $\mathrm{D}$, generating an $\mathrm{N}$-terminal fragment that oligomerizes to form pores on the cell membrane and cause programmed cell death known as pyroptosis [8-11]. In previous studies, NLRP3 can be activated by different mediators such as DAMPs and/or PAMPs in vivo, and NLRP3 plays a crucial role in the development of IRI in various organs [7, 12, 13]. Therefore, inhibiting NLRP3 inflammasome activation may play a protective role in IRI.

Mesenchymal stromal cells (MSCs) are a group of stem cells derived from the embryonic mesoderm, which can differentiate into various kinds of cells $[4,14,15]$. MSCs have the proliferative, pluripotent and immunomodulatory potentials that can help repair damaged tissues and improve tissue microenvironment [16]. Accumulating evidence have demonstrated that MSCs treatment could alleviate IRI in various 
organs by inhibiting intensive inflammation, apoptosis, generations of oxidative stress, ROS, as well as immune over-reactivity [17, 18].

Interleukin 37 (IL-37) is a novel cytokine that recently characterized a member in the IL-1 family, which plays a key role in limiting excessive and runaway inflammatory responses via suppressing both innate and adaptive immunity [19-21]. It has been demonstrated that a knockdown of endogenous IL-37 in human peripheral blood mononuclear cells, which results in increased production of several pro-inflammatory cytokines [22]. Human IL-37 transgenic mice are protected against metabolic syndrome, systemic inflammation reaction, DSS-induced colitis, and acute myocardial infarction. As an immunomodulatory factor, IL-37 has been tested in IRI models such as myocadial and renal IRI [23, 24].

Although MSCs and IL-37 could protect organs against IRI, their roles were limited for many reasons $[25,26]$. Thus, it is necessary to combine them together to enhance their therapeutic effects. In this study, we successfully conducted IL-37 gene modified MSCs and investigated their better effects on prevention of intestinal IRI and explored potential mechanisms.

\section{Methods}

\section{Animals}

Male Sprague-Dawley (SD) rats weighing 250-300g were purchased from China National Institute for Food and Drug Control and received a standard temperature, diet and water in the Animal Care Facility of Tianjin General Surgery Institute. All 
animal experimental operations were approved by the Institute of Animal Care and Use Committee at Tianjin Medical University and performed in accordance with the Guide for the Care and Use of Laboratory Animals.

\section{Isolation and culture of MSCs}

MSCs were prepared according to the protocol described previously [4]. Briefly, in order to harvest the adipose tissue surrounding the inguen, rats were sacrificed and soaked in $75 \%$ alcohol for 10 mins. Then, $200-300$ ul sterile PBS was added to every $0.5 \mathrm{~g}$ adipose tissue to prevent dehydration. The tissue was cut into $<1 \mathrm{~mm}^{3}$ pieces, followed by the addition of type I collagenase solution $(1 \mathrm{mg} / \mathrm{mL}$, Solarbio, Beijing, China). The tubes with contents were placed on a shaker and incubated with constant agitation for 60 minutes $\left(37^{\circ} \mathrm{C}, 200 \mathrm{rpm}\right)$. Next, an equal volume of serum-containing medium was added to terminate digestion. After that the solution went through centrifuge (1800rpm, 5min), discarded the supernatant and wash for twice by PBS. Lastly, cells were inoculated in $15 \%$ fetal bovine serum (FBS, Hyclone, Touranga, New Zeland) containing $\alpha$-MEM medium (Hyclone, Touranga, New Zeland) and 1\% penicillin/streptomycin (Solarbio, Beijing, China) and subcultured after 7-10 days. The morphology and surface markers were detected to identify MSCs.

\section{Preparation and identification of IL-37-MSCs}

The construction of vector expressing IL-37 (NM_014439, IL-37 isoform b, which is best characterized, Ubi-MCS-3FLAG-SV40-EGFP-IRES-puromycin), sequencing 
(results are shown in the supplement) and lentiviral packaging were supported by Genechem. Inc., Shanghai China. We conducted lentiviral transfection of MSCs according to the protocol provided by Genechem. Inc. (the vector that lacks IL-37 was used as control). Lentiviral transfection is done in a suitable multiplicity of infection (MOI=200, ratio of lentivirus to cell number) under biological safety cabinet. The cells then were observed under inverted fluorescence microscope at post-transfection 72 hours and IL-37 expression was identified by immunofluorescence (IF, details are shown in methods of immunofluoscence).

Furthermore, IL-37-MSCs were subjected to drug screening using $1 \mathrm{ug} / \mathrm{mL}$ puromycin (Solarbio, Beijing, China) containing medium to obtain high purity IL-37-MSCs, and flow cytometry was performed before treatment to ensure cell purity and quality.

\section{Flow cytometry analysis}

The markers of MSCs and IL-37-MSCs positive rate were analyed by using flow cytometric analysis. In brief, MSCs were stained with fluorescent antibodies, including anti-CD29-FITC, anti-CD45-PE, anti-79a-PE, anti-CD90-FITC ( $e$ Biosciences, San Diego, USA) according to the manufacturer's instruction. The Flowjo software was used to analyze the percentages of various markers of MSCs.

\section{Immunofluorescence}

We used IF technology to identify IL-37 expression in IL-37-MSCs to ensure 
successful transfection, MSCs (negative control lentiviral transfection which express GFP while lacking IL-37) were used as control. Briefly, first of all, MSCs were cultured in the polylysine pretreated slides overnight. Then, cells were fixed by $4 \%$ paraformaldehyde (PFA) for half an hour and treated with $0.1 \%$ Triton-X (Solarbio, Beijing, China) for 2 minutes. After that, cells were treated with 5\% BSA for 30 minutes to reduce non-specific combination. Next, anti-IL-37 antibody (dilution at 1:250, Abcam, Cambridge, UK) was added to the slides for night, after washed three times by PBS, the cy3-conjugated goat anti-rabbit secondary antibody was added for half an hour and washed three times again. DAPI (Thermo Fisher Scientific, Waltham, USA) was added dropwise before coverslip. Lastly, slides were observed under fluorescence microscope.

\section{Experimental Groups}

To test the effects of IL-37-MSCs on protecting against intestinal IRI, SD rats were randomly assigned to five groups ( $\mathrm{n}=8$ each group): sham control group, IL-37-MSC-treated IRI group, MSC-treated IRI group, recombinant IL-37 (rIL-37)-treated (Peprotech, New Jersey, US) IRI group and untreated IRI group. In the beginning, rats got intraperitoneal injection of pentobarbital sodium $(50 \mathrm{mg} / \mathrm{kg})$, heating pad was used to maintain their temperature and abdomen hair was removed. After abdominal disinfection, SMA was isolated by a midline incision into the abdominal cavity. In the sham control group, rats were only operated by opening and closing the abdomen, without clipping the SMA. In other groups, the roof of SMA 
was clipped for $30 \mathrm{~min}$ then recovered reperfusion. After restoring the blood supply and closing the incision, $2 \times 10^{6} \mathrm{IL}-37-\mathrm{MSCs}, 2 \times 10^{6} \mathrm{MSCs}$, $2 \mathrm{ug}$ rIL-37 (according to our pre-experimental results, $2 \mathrm{ug}$ each rat was used in this study) and $1 \mathrm{~mL}$ PBS were separately injected into tail vein. Rats were maintained by continuous monitoring with a temperature-controlled self-regulated heating system after operation. After reperfusion for 72 hours, all rats were sacrificed for IRI assessment.

\section{Tracing of Infused IL-37-MSCs}

As IL-37-MSCs expressing GFP protein, we could detect fluorescent protein expression in the damaged tissue to identify whether IL-37-MSCs could migrate to the injured intestine. Ileums were embedded in OCT for frozen section and then slides were observed under fluorescent microscope to detect GFP expression.

\section{Histology}

To evaluate the impact of IL-37-MSCs transplantation on the severity of intestinal IRI, the ileum was collected for assessment [27]. Briefly, intestine specimens were fixed in $10 \%$ formalin for 72 hours, then embedded in paraffin with correct orientation position of the crypt to villus axis and sectioned at $5 \mu \mathrm{m}$ for hematoxylin and eosin $(\mathrm{H} \& \mathrm{E})$ staining to assess the severity of injury site. Chiu's score grading was used as standard: 0 , normal villi of the small intestinal mucosa; 1 , Gruenhagen's space under the intestinal mucosal epithelium in the villus axis, often accompanied by capillary congestion; 2, intestinal mucosal epithelium elevation from the intrinsic 
membrane and expansion of the intestinal subepithelial space; 3, large intestinal mucosal epithelium elevation, villus lodging to both sides, part of the villus shed; 4 , villus and lamina detachment, bare capillaries dilate, an increase in the composition of lamina propria cell components; and 5, lamina propria is digested, bleeding or ulcers form $[14,28]$. The slides from each sample and each slide with five fields at a magnification $\times 100$ were observed by a professional pathologist, and the average scores of each group were calculated. The tests were random and double-blind.

\section{Enzyme-linked Immunosorbent Assay (ELISA)}

D-Lactic acid (D-Lac) and Diamine oxidase (DAO) in the serum were used to assess the gut barrier function [29]. IL-1 $\beta$ in the tissue and serum were uesd to assess the local and systemic inflammation activity. DAO, D-Lac ELISA kits (Senbeijia, Nanjing, China) and IL-1 $\beta$ ELISA kit (DAKEWE, Shenzhen, China) were used to evaluate the levels in the serum or tissue. All operations conducted according to the manufacturer's recommended protocol.

\section{Real-Time PCR}

Total RNA was obtained from ileum tissue by using RNAprep Pure Tissue Kit (Tiangen, Beijing, China) and reverse-transcribed were performed by FastQuant RT Super Mix (Tiangen, Beijing, China), mRNA expression was quantified by 2x SYBR Green qPCR Master Mix (Bimake, Houston, TX, USA). In the reaction system, $\beta$-actin was used as an internal normalizing gene, and mRNA expression was 
analyzed by comparative threshold cycle (Ct value) of $2^{-\Delta \Delta \mathrm{ct}}$. The primer sequences used were as follows, $\beta$-actin forward: 5'-GTTG ACAT CCGT AAAG AC-3', reverse: 5'-TGGA AGGT GGAC AGTG AG-3', TNF- $\alpha$ forward: 5'ACAC ACGA GACG CTGA AGTA-3', reverse: 5'-GGAA CAGT CTGG GAAG TCT-3', IL-6 forward: 5'-CTCA TTCT GTCT CGAG CCCA-3', reverse: 5'- CTGT GAAG TCTC CTCT CCGG -3'.

\section{Western-Blot}

Ileum tissues were homogenized and total protein was extracted by RIPA lysis mixed with PMSF (Solarbio, Beijing, China). Then 50ug of protein per sample was subjected to $7.5 \%, 10 \%$ or $15 \%$ sodium dodecyl sulfate polyacrylamide gel electrophoresis (SDS-PAGE, Solarbio, Beijing, China). After overnight incubation at $4{ }^{\circ} \mathrm{C}$ with anti-NLRP3 antibody (dilution at 1:1000, abcam, Cambridge, UK), anti-caspase-1 antibody (dilution at 1:300, Santa cruz, Oregon, USA), anti-IL-1 $\beta$ antibody (dilution at 1:600, Bioss, Beijing, China), anti-IL-18 antibody (dilution at 1:600, Bioss, Beijing, China), anti- $\beta$-actin antibody (dilution at 1:2000, Servicebio, Wuhan, China) and anti-HSP-90 antibody (dilution at 1:1000, Santa cruz, Oregon, USA), the membranes with blotted proteins were then incubated with HRP-conjugated goat anti-rabbit secondary antibody (dilution at 1:2000, CST, Boston, USA) or rat anti-mouse secondary antibody (dilution at 1:2000, Servicebio, Wuhan, China) for an hour at room temperature. After washing three times with TBST, the electrochemiluminescence solution (ECL, Millipore, Massachusetts, USA) was added 
to the membranes, finally exposed to the exposure machine (ChemiScope series, Clinx Science Instruments Co., Ltd), the images were recorded and then analyzed.

\section{Statistics}

GraphPad Prism (version:8) was used to perform all statistical analysis. Data were expressed as mean \pm SD and one-way analysis of variance (ANOVA) was used for group comparisons. Throughout the text, figures, and legends, the following terminology is used to denote statistical significance: *, $p<0.05 ; * *, p<0.01$; ***, $p<0.001$.

\section{Results}

\section{Characterization of MSCs and IL-37-MSCs}

As shown in Figure 1a, MSCs showed spindle-shaped, fibroblast-like morphology after passage 2 and exhibited colony-forming ability. At passage 3, MSCs were detected and demonstrated high expression of CD29 and CD90, while lacking CD45 and CD79a expressions (Figure 1b). As shown in Figure 1c, at 72 hours post-transfection, the expression of GFP fluorescent protein was observed under a fluorescence microscope. In addition, IL-37 expression was found in IL-37-MSCs but not in MSCs, which met our expectations. After passing through the puromycin drug screening, the GFP positive rate was above $99.8 \%$ measured by flow cytometry before cell treatment which met our needs (Figure 1d). 


\section{Transplanted IL-37-MSCs could migrate to the injured tissue in vivo}

To investigate whether IL-37-MSCs could migrate to damaged intestine through the intravenous injection, intestine tissues were fixed by OCT for frozen section to detect the GFP expression. As shown in Figure 1e, GFP expression was positive in IL-37-MSC-treated and MSC-treated groups while sham control group not, which meant that infused IL-37-MSCs and MSCs could migrate to the injured tissue.

\section{IL-37-MSCs significantly ameliorated pathological IRI damage of intestine}

Chiu's Score was used to assess the tissue damage. As shown in Figure 2a, no obvious abnormal tissue changes were observed in the sham group. However, intestinal injury in untreated group was severe, which was characterized by villus damage, epithelial necrosis, subendothelial hemorrhage and neutrophils infiltration. As expected, intestinal damage scores following IRI were significantly improved by the mere use of MSCs and rIL-37, furthermore improved by IL-37-MSCs administration (IL-37-MSC-treated $v s$. MSC-treated, $p<0.01$; IL-37-MSC-treated $v s$. rIL-37-treated, $p<0.01$, Figure 2b).

\section{IL-37-MSCs improved intestinal barrier function following intestinal IRI}

To determine whether IL-37-MSCs could attenuate intestinal IRI, we measured the serum DAO and D-Lac, which represented the intestine barrier function as described above. As shown in Figure 3, it was found that DAO and D-Lac showed highest levels in untreated group, which reduced by different treatments. Compared with MSCs- or 
rIL-37 treated group, IL-37-MSCs significantly decreased DAO and D-Lac levels in the serum (DAO level: IL-37-MSC-treated vs. MSC-treated, $p<0.01$; IL-37-MSC-treated vs. rIL-37-treated, $p<0.01$, Figure 3a; D-Lac level: IL-37-MSC-treated vs. MSC-treated, $p<0.05$; IL-37-MSC-treated vs. rIL-37-treated, $p<0.01$, Figure 3b), which indicated that IL-37-MSCs could effectively improve intestine barrier function following intestinal IRI.

\section{IL-37-MSCs decreased local and systemic inflammation reactivity cytokine IL-1ß}

We used cytokine IL-1 $\beta$ level to evaluate local and systemic inflammation reactivity. As shown in Figure 4, local and systemic cytokine IL-1 $\beta$ was significantly increased in untreated IRI group. In addition, IL-37-MSCs treatment significantly decreased local and systemic IL-1 $\beta$ level following IRI compared with MSCs and/or rIL-37 treatment (local IL-1 $\beta$ level: IL-37-MSC-treated vs. MSC-treated, $p<0.05$; IL-37-MSC-treated vs. rIL-37-treated, $p<0.001$, Figure 4a. systemic IL-1 $\beta$ level: IL-37-MSC-treated vs. MSC-treated, $p<0.05$; IL-37-MSC-treated vs. rIL-37-treated, $p<0.01$, Figure 4b).

Infusion of IL-37-MSCs decreased the NLRP3 inflammasome activation and relative protein expressions

NLRP3 played an important role in the development of various diseases, and inhibiting NLRP3 activation could effectively attenuate intestinal IRI. In this study, to detect whether IL-37-MSCs could effectively inhibit NLRP3 inflammasome 
activation, we performed Western Blot to detect NLRP3 and its downstream protein expressions. As shown in Figure 5a, NLRP3 expression in IL-37-MSC-treated group was significantly lower than that in MSC-treated and/or rIL-37-treated groups (IL-37-MSC-treated vs. MSC-treated, $p<0.01$; IL-37-MSC-treated vs. rIL-37-treated, $p<0.01)$. Cleaved caspase- 1 expressions in each group were in accordance with NLRP3 expressions, compared with sole MSCs or rIL-37 treatment, IL-37-MSCs markedly decreased cleaved caspase-1 expression (IL-37-MSC-treated vs. MSC-treated, $p<0.001$; IL-37-MSC-treated vs. rIL-37-treated, $p<0.001$, Figure 5b). As a result, IL-1 $\beta$ and IL-18 protein expressions in tissues were also in accordance with NLRP3 expression, IL-1 $\beta$ and IL-18 protein expressions in IL-37-MSC-treated group were lower than those in MSC-treated or rIL-37 treated group (IL-1 $\beta$ expression: IL-37-MSC-treated vs. MSC-treated, $p<0.001$; IL-37-MSC-treated $v s$. rIL-37-treated, $p<0.001$, Figure 5c. IL-18 expression: IL-37-MSC-treated $v s$. MSC-treated, $p<0.001$; IL-37-MSC-treated vs. rIL-37-treated, $p<0.001$, Figure 5d).

\section{IL-37-MSCs treatment decreased local IL-1ß and IL-18 relative} pro-inflammatory cytokines mRNA expression

Proinflammatory cytokines count in the intestinal IRI. Hence, decreasing these proinflammatory cytokines expressions may contribute to alleviate tissue injury. As shown in Figure 6, the TNF- $\alpha$ and IL-6 mRNA expressions in IL-37-MSC-treated were obviously lower than MSC-treated or rIL-37-treated IRI groups (TNF- $\alpha$ mRNA expression: IL-37-MSC-treated vs. MSC-treated, $p<0.05$; IL-37-MSC-treated $v s$. 
rIL-37-treated, $p<0.01$, Figure 6a. IL-6 mRNA expression: IL-37-MSC-treated $v s$. MSC-treated, $p<0.05$; IL-37-MSC-treated vs. rIL-37-treated, $p<0.01$, Figure 6b).

\section{Discussion:}

Our study, which investigated the therapeutic effects of IL-37-MSCs treatment on inhibiting intestinal IRI, provided several preclinical implications of IL-37 and gene-modified MSCs. First, as compared with the sham control group, tissue damage scores (H\&E assessment) were remarkably enhanced in animals with IRI. As expected, the parameter was significantly suppressed in animals with IRI after MSCs or rIL-37 treatment and further notably decreased following IL-37-MSCs transplantation therapy. Second, intestinal barrier function as measured by DAO and D-Lac were preserved in a manner consistent with tissue damage scores in all groups. Third, not only local but systemic inflammatory cytokine IL-1 $\beta$ level was markedly attenuated in all treatment groups following IRI. Then, NLRP3-mediated pro-inflammatory signaling pathway was found to be upregulated in untreated IRI group, which was significantly suppressed following MSCs, rIL-37 and IL-37-MSCs treatments, suggesting the multifactorial nature of underlying mechanisms involved in intestinal IRI, for which IL-37-MSCs demonstrated much more powerful effects than MSCs or rIL-37 alone in attenuation of intestinal IRI.

The intestinal tract was one of the organs that were pretty sensitive to ischemia. In addition, intestinal IRI usually lead to systemic inflammatory responses (SIRs) and multiple organ dysfunction syndromes (MODs), which was one of the most high 
morbidity and mortality diseases in the clinic [3]. Various inflammatory cells involved in the whole process of disease, such as mucosal cells, macrophages, neutrophils, endothelial cells, etc., which were responsible for the release of different cytokines, chemokines and free radicals following intestinal IRI. Therefore, we believed that the mechanism of ischemia reperfusion injury is closely related to the release of ROS after ischemia or hypoxia [7]. Excessive ROS activated NLRP3 inflammasome and eventually lead to the excessive release of proinflammatory cytokines IL-1 $\beta$ and IL-18 [7]. The dysregulation of NLRP3 inflammasome could cause excessive inflammation and play a pivotal role in many human diseases. Previous studies have demonstrated that NLRP3 inflammasome involved in several ischemia reperfusion injury organ such as heart, liver, kidney and so on. Interestingly, little has been reported about the role of NLRP3 in intestinal ischemia-reperfusion injury until Wang et al. identified that NLRP3 actually count in intestinal IRI [30]. Several studies suggested that targeting inhibiting NLRP3 inflammasome activation could alleviate ischemia reperfusion injury occurring in different organs [7, 12, 13, 31, 32]. Therefore, it was really a great way to treat intestinal IRI by inhibiting NLRP3 inflammasome activation.

As we all know, cell therapy strategy has been reported to be a strongly effective way in the treatment of different diseases $[16,18,33,34]$. MSCs act as a kind of adult stem/stromal cells that could differentiate into specific tissues cells induced by local microenvironment when they were damaged, and could secrete various cytokines. Previous studies have demonstrated that MSCs, especially those adipose-derived, 
possess anti-inflammatory and immunomodulatory functions. Intriguingly, MSCs and IL-37 both could inhibit NLRP3 inflammasome activation, but different mechanisms were involved as reported. MSCs have been noted for ischemia-reperfusion injury and good results have been obtained, which could decrease NLRP3 activation via clearing excessive ROS as reported [18]. However, as described above, IL-37 plays an important anti-inflammatory and immunomodulatory capacity in a variety of inflammatory and autoimmune diseases. In addition, IL-37 in vivo could inhibit NLRP3 activation also be mentioned in colitis and LPS-induced disease [19, 22, 35-37]. In recent study, Rudloff et al reported that IL-37 significantly suppress inflammasome activity in vivo to ameliorate inflammasome-driven diseases, which could corroborate our study results to some extent [38].

However, either mesenchymal stromal cells or IL-37 showed limited roles to relieve intestinal IRI $[25,26,39]$. Thus, it was very important to find a way to enhance the effects of MSCs. During the past decades, remarkable progresses have been made in the area of gene engineered MSCs bases therapy. Based on these points, we utilized MSCs as a vehicle to drive IL-37 and further release IL-37 in vivo (result is demonstrated in supplement that IL-37 expression in IL-37-MSCs-treated group was higher than MSCs-treated group) to help better inhibit NLRP3 inflammasome activation to alleviate damage, thereby enhancing the effects of MSCs in the intestinal IRI model.

It is the first time to demonstrate that IL-37 could play a protective role in the intestinal IRI and illustrated IL-37 gene modification could enhance the therapeutic 
effects of MSCs in the ischemia reperfusion injury. However, many mechanisms are involved in the IL-37-MSCs treatment in intestinal IRI, and more specific mechanisms remains to be explored.

\section{Conclusion:}

In conclusion, IL-37 gene modification could enhance the therapeutic effects of MSCs. IL-37-MSCs improved intestine barrier function, improved injured tissue microenvironment and inhibited NLRP3-mediated signaling pathway, which exert a much better protective role in intestinal IRI than MSCs. NLRP3-related signaling pathways could be related to the process of IL-37-MSC mediated protection. IL-37-MSCs treatment acted as an effective tool to protect intestine against IRI.

\section{Abbreviations}

MSCs: mesenchymal stromal cells;

rIL-37: recombinant interleukin 37

IL-37-MSCs: interleukin 37 gene-modified mesenchymal stromal/stem cells;

IRI: ischemia reperfusion injury;

SMA: superior mesentric artery;

NOD-like: nucleotide-binding oligomerization domain-like;

NLRP3: NOD-like receptor family, pyrin domain protein 3;

FBS: fetal bovine serum;

PBS: Phosphate buffer saline; 
H\&E: hematoxylin and eosin;

DAO: diamine oxidase;

D-Lac: D-Lactate;

IL-1 $\beta$ : interleukin $1 \beta$;

IL-18: interleukin 18.

\section{Declarations}

\section{Ethical approval and consent to participate}

All the experiments were performed according to the Chinese Council on Animal

Care guidelines and the basis of protocols approved by the Animal Care and Use Committee of Tianjin Medical University (Tianjin, China) (IRB2019-YX-001).

\section{Consent for publication}

Not applicable.

\section{Availability of data and material}

The dataset supporting the conclusions of this article is included within the article.

\section{Competing interests}

The authors declare that they have no conflict of interests. 


\section{Funding}

This work was supported by grants to H.W. from National Natural Science Foundation of China (No. 81273257 and 81471584), Tianjin Application Basis and

Cutting-Edge Technology Research Grant (No. 14JCZDJC35700), Li Jieshou Intestinal Barrier Research Special Fund (No. LJS_201412), Natural Science Foundation of Tianjin (No. 18JCZDJC35800), Tianjin Medical University Talent Fund, and Tianjin Research Innovation Project for Postgraduate Students to Dejun Kong (No. 2019YJSS184).

\section{Authors' contributions}

Dejun Kong, Yonghao $\mathrm{Hu}$ and Xiang Li are co-first authors on this paper. Dejun Kong designed and carried out the research, analyzed the data and drafted the manuscript; Yonghao $\mathrm{Hu}$ and Xiang Li designed and carried out the research and polished the manuscript; Dingding Yu, Hongyue Li carried out the research; Yiming Zhao and Yafei Qin polished the manuscript; Baoren Zhang, Wang Jin, Bo Wang, Hongda Wang and Guangming Li performed the research; H. Wang conceived of the study, participated in research design and coordination, and helped to draft and edit the manuscript. All authors read and approved the final manuscript.

\section{Acknowledgements}

Not applicable. 


\section{References:}

1. Yan, X.T., et al., The HO-1-expressing bone mesenchymal stem cells protects intestine from ischemia and reperfusion injury. BMC Gastroenterol, 2019. 19(1): p. 124.

2. Chassin, C., et al., MicroRNA-146a-mediated downregulation of IRAKI protects mouse and human small intestine against ischemia/reperfusion injury. EMBO Mol Med, 2012. 4(12): p. 1308-19.

3. Acosta, S. and M. Bjorck, Modern treatment of acute mesenteric ischaemia. Br J Surg, 2014. 101(1): p. e100-8.

4. Chang, C.L., et al., Protective effect of melatonin-supported adipose-derived mesenchymal stem cells against small bowel ischemia-reperfusion injury in rat. J Pineal Res, 2015. 59(2): p. 206-20.

5. $\mathrm{Hu}, \mathrm{C}$., et al., Modulating autophagy in mesenchymal stem cells effectively protects against hypoxia- or ischemia-induced injury. Stem Cell Res Ther, 2019. 10(1): p. 120.

6. Mridha, A.R., et al., NLRP3 inflammasome blockade reduces liver inflammation and fibrosis in experimental NASH in mice. J Hepatol, 2017. 66(5): p. 1037-1046.

7. Minutoli, L., et al., ROS-Mediated NLRP3 Inflammasome Activation in Brain, Heart, Kidney, and Testis Ischemia/Reperfusion Injury. Oxid Med Cell Longev, 2016. 2016: p. 2183026.

8. Guo, H., J.B. Callaway, and J.P. Ting, Inflammasomes: mechanism of action, role in disease, and therapeutics. Nat Med, 2015. 21(7): p. 677-87.

9. Liu, X., et al., Inflammasome-activated gasdermin D causes pyroptosis by forming membrane pores. Nature, 2016. 535(7610): p. 153-8.

10. Broz, P., Immunology: Caspase target drives pyroptosis. Nature, 2015. 526(7575): p. 642-3.

11. Shi, J., et al., Cleavage of GSDMD by inflammatory caspases determines pyroptotic cell death. Nature, 2015. 526(7575): p. 660-5. 
12. Qiu, Z., et al., NLRP3 Inflammasome Activation-Mediated Pyroptosis Aggravates Myocardial Ischemia/Reperfusion Injury in Diabetic Rats. Oxid Med Cell Longev, 2017. 2017: p. 9743280.

13. Ito, M., et al., Bruton's tyrosine kinase is essential for NLRP3 inflammasome activation and contributes to ischaemic brain injury. Nat Commun, 2015. 6: p. 7360.

14. Geng, Y., et al., Synergistic Effects of Electroacupuncture and Mesenchymal Stem Cells on Intestinal Ischemia/Reperfusion Injury in Rats. Inflammation, 2016. 39(4): p. 1414-20.

15. Li, J., W. Liu, and W. Yao, Immortalized Human Bone Marrow Derived Stromal Cells in Treatment of Transient Cerebral Ischemia in Rats. J Alzheimers Dis, 2019. 69(3): p. 871-880.

16. Wang, Y., et al., Plasticity of mesenchymal stem cells in immunomodulation: pathological and therapeutic implications. Nat Immunol, 2014. 15(11): p. 1009-16.

17. Sun, P., et al., Human endometrial regenerative cells attenuate renal ischemia reperfusion injury in mice. J Transl Med, 2016. 14: p. 28.

18. Nakajima, D., et al., Mesenchymal stromal cell therapy during ex vivo lung perfusion ameliorates ischemia-reperfusion injury in lung transplantation. J Heart Lung Transplant, 2019.

19. Nold, M.F., et al., IL-37 is a fundamental inhibitor of innate immunity. Nat Immunol, 2010. 11(11): p. 1014-22.

20. Nold-Petry, C.A., et al., IL-37 requires the receptors IL-18Ralpha and IL-1R8 (SIGIRR) to carry out its multifaceted anti-inflammatory program upon innate signal transduction. Nat Immunol, 2015. 16(4): p. 354-65.

21. Banchereau, J., V. Pascual, and A. O'Garra, From IL-2 to IL-37: the expanding spectrum of anti-inflammatory cytokines. Nat Immunol, 2012. 13(10): p. 925-31.

22. Conti, P., et al., Critical role of inflammatory mast cell in fibrosis: Potential therapeutic effect of IL-37. Cell Prolif, 2018. 51(5): p. e12475. 
23. Yang, Y., et al., IL-37 inhibits IL-18-induced tubular epithelial cell expression of pro-inflammatory cytokines and renal ischemia-reperfusion injury. Kidney Int, 2015. 87(2): p. 396-408.

24. $\mathrm{Wu}, \mathrm{B}$., et al., Interleukin-37 ameliorates myocardial ischaemia/reperfusion injury in mice. Clin Exp Immunol, 2014. 176(3): p. 438-51.

25. Huang, L., et al., Interleukin-13 Gene Modification Enhances Grafted Mesenchymal Stem Cells Survival After Subretinal Transplantation. Cell Mol Neurobiol, 2019.

26. Shomali, N., et al., Mesenchymal stem cells as carrier of the therapeutic agent in the gene therapy of blood disorders. J Cell Physiol, 2019.

27. Gonzalez, L.M., A.J. Moeser, and A.T. Blikslager, Animal models of ischemia-reperfusion-induced intestinal injury: progress and promise for translational research. Am J Physiol Gastrointest Liver Physiol, 2015. 308(2): p. G63-75.

28. Cheng, C.H., et al., Ischemic postconditioning attenuate reperfusion injury of small intestine: impact of mitochondrial permeability transition. Transplantation, 2013. 95(4): p. 559-65.

29. Cosse, C., et al., Procalcitonin and intestinal ischemia: a review of the literature. World J Gastroenterol, 2014. 20(47): p. 17773-8.

30. Wang, Z., et al., Autophagy Induction Ameliorates Inflammatory Responses in Intestinal Ischemia-Reperfusion Through Inhibiting NLRP3 Inflammasome Activation. Shock, 2019. 52(3): p. 387-395.

31. Inoue, Y., et al., NLRP3 regulates neutrophil functions and contributes to hepatic ischemia-reperfusion injury independently of inflammasomes. $\mathrm{J}$ Immunol, 2014. 192(9): p. 4342-51.

32. Nazir, S., et al., Cytoprotective activated protein $C$ averts Nlrp3 inflammasome-induced ischemia-reperfusion injury via mTORC1 inhibition. Blood, 2017. 130(24): p. 2664-2677.

33. Fazeli, Z., et al., Mesenchymal Stem Cells (MSCs) Therapy for Recovery of Fertility: a Systematic Review. Stem Cell Rev, 2018. 14(1): p. 1-12. 
34. Green, L.J., et al., Adipose-derived stem cells promote survival, growth, and maturation of early-stage murine follicles. Stem Cell Res Ther, 2019. 10(1): p. 102.

35. Ballak, D.B., et al., IL-37 protects against obesity-induced inflammation and insulin resistance. Nat Commun, 2014. 5: p. 4711.

36. Luo, P., et al., IL-37b alleviates inflammation in the temporomandibular joint cartilage via IL-1R8 pathway. Cell Prolif, 2019: p. e12692.

37. Yang, Z., et al., Role of IL-37 in Cardiovascular Disease Inflammation. Can J Cardiol, 2019. 35(7): p. 923-930.

38. Rudloff, I., et al., Parsing the IL-37-Mediated Suppression of Inflammasome Function. Cells, 2020. 9(1).

39. Wang, W.Q., et al., IL-37b gene transfer enhances the therapeutic efficacy of mesenchumal stromal cells in DSS-induced colitis mice. Acta Pharmacol Sin, 2015. 36(11): p. 1377-87. 


\section{Figure legends:}

Figure 1. IL-37-MSCs and MSCs could migrate to the injured tissue. The morphology of MSCs. Passage 0,1, 2 and 3 of MSCs were showed above (Figure 1a). FACS analysis of MSCs surface marker was shown in Figure 1b, surface expressions of CD29, CD45, CD79a, CD90 were detected. The IL-37 and GFP expressions were detected in IL-37-MSCs and MSCs (Figure 1c). As shown in Figure 1d, IL-37-MSCs GFP positive rate was detected, positive rate was above $99.8 \%$ which met our needs. IL-37-MSC-treated and MSC-treated intestine exhibited significant GFP expression while sham group not as shown in Figure 1e, which suggested IL-37-MSCs and MSCs could migrate to the injured tissue.

Figure 2. IL-37-MSCs significantly ameliorated pathological intestine damage

following IRI. IL-37-MSCs could Microscopic findings illustrated the architecture of ileum by 72 hours after reperfusion, the damage score was assessed according to Chiu's Score. As shown in Figure 2a, compared with sham group, untreated group demonstrated severe damage such as inflammatory cells infiltration, hemorrhage and ulcer. However, as shown in Figure 2b IL-37-MSC-treated IRI group showed more significant therapeutic effects compared with MSC-treated and rIL-37-treated groups. The data suggested IL-37-MSCs provide a better protective role in intestinal IRI. Data shown were representative and the $p$ value was determined by one-way ANOVA. * $p<0.05, * * p<0.01, * * * p<0.001$. 
Figure 3. IL-37-MSCs could effectively improve intestinal barrier function following IRI. DAO and D-Lac were used to assess the gut barrier function. Serum samples were collected from sham, IL-37-MSC-treated, MSC-treated, rIL-37-treated and untreated IRI groups. In comparison with MSC-treated and rIL-37-treated IRI groups, IL-37-MSCs significantly reduced serum levels of DAO and D-Lac, which meant IL-37-MSCs remarkedly improved gut barrier function. Data shown were representative and the $p$ value was determined by one-way ANOVA. * $p<0.05$, ** $p<0.01, * * * p<0.001$

Figure 4. IL-37-MSCs could significantly decrease local and systemic inflammation activity. Local and systemic inflammation activity was assessed by IL-1 $\beta$ level in the local tissue and serum. Compared with MSCs and rIL-37 treatment, IL-37-MSCs significantly alleviate the local and system inflammation activity. The $p$ value was determined by one-way ANOVA. $* p<0.05, * * p<0.01, * * * p<0.001$.

\section{Figure 5. IL-37-MSCs treatment decreased NLRP3 and relative proteins}

expressions. As shown in Figure 5a, compared with MSCs and IL-37 treatment, IL-37-MSCs significantly decreased the NLRP3 activation. In parallel with NLRP3 expression, cleaved caspase-1, IL-1 $\beta$ and IL-18 proteins expressions were significantly decreased following IL-37-MSCs treatment compared with MSCs and/or rIL-37 treatments (Figure 5b,c \& d), which suggested that IL-37-MSCs could inhibit NLRP3-mediated signaling pathway. Data shown were representative above and the $p$ 
value was determined by one-way ANOVA. * $p<0.05, * * p<0.01, * * * p<0.001$.

\section{Figure 6. IL-37-MSCs transplantation decreased IL-6 and TNF- $\alpha$ mRNA}

expression. IL-1 $\beta$ and IL-18 are key proinflammatory cytokines, and as their downstream pro-inflammation molecules, IL-6 and TNF- $\alpha$ play a pivotal role in inflammatory reactivity. As shown in Figure 6, intestine IL-6 and TNF- $\alpha$ mRNA expressions in IL-37-MSC-treated group were significantly decreased compared with MSCs and rIL-37 treated group. The $p$ value was determined by one-way ANOVA. $* p<0.05, * * p<0.01, * * * p<0.001$. 


\section{Figures}

a
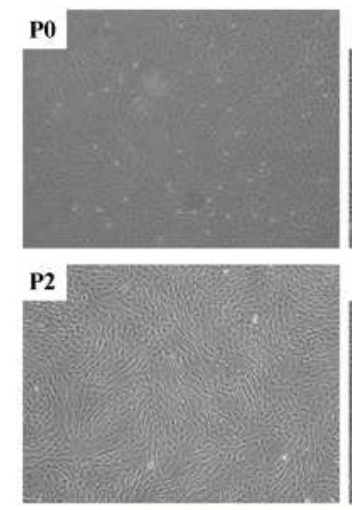

c

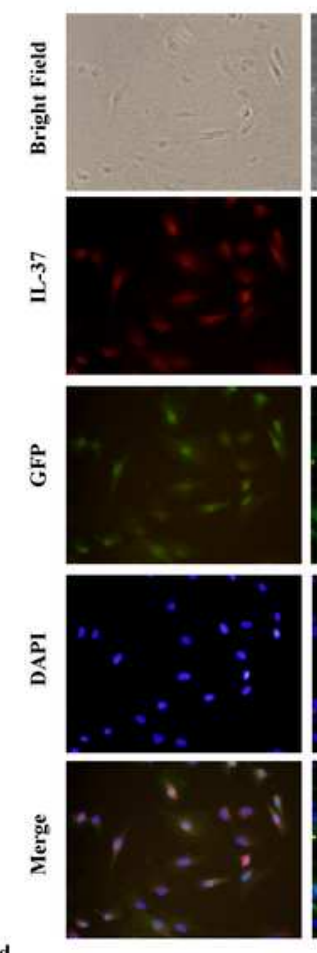

d
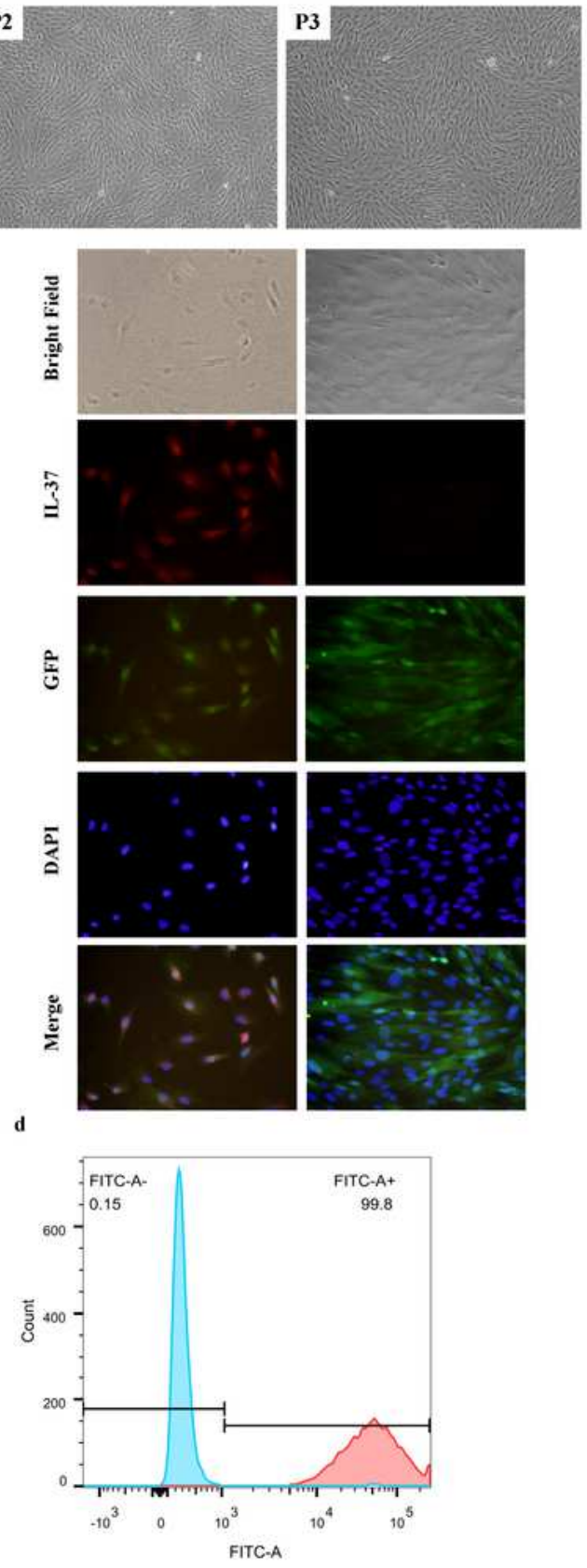

b

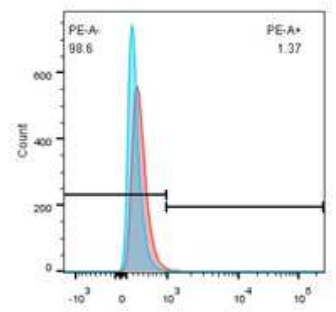

CD45

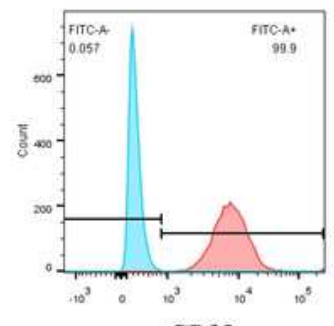

CD29

e

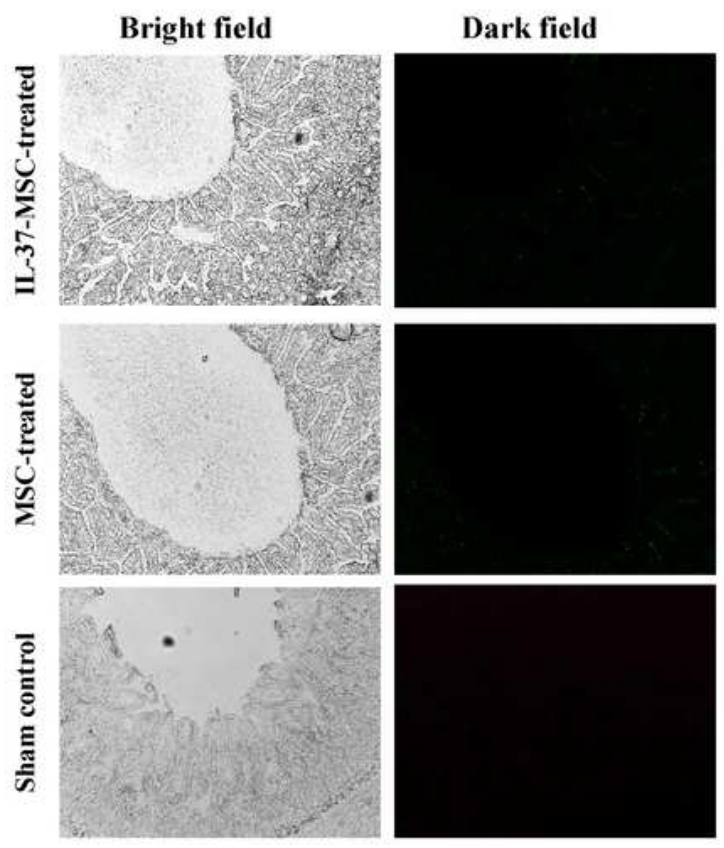

Figure 1

\section{Figure 1}

IL-37-MSCs and MSCs could migrate to the injured tissue. The morphology of MSCs. Passage 0, 1, 2 and 3 of MSCs were showed above (Figure 1a). FACS analysis of MSCs surface marker was shown in Figure $1 \mathrm{~b}$, surface expressions of CD29, CD45, CD79a, CD90 were detected. The IL-37 and GFP expressions were 
detected in IL-37-MSCs and MSCs (Figure 1c). As shown in Figure 1d, IL-37-MSCs GFP positive rate was detected, positive rate was above $99.8 \%$ which met our needs. IL 37 MSC treated and MSC treated intestine exhibited significant GFP expression while sham group not as shown in Figure $1 \mathrm{e}$, which suggested IL 37 MSCs and MSCs could migrate to the injured tissue. Figure

a
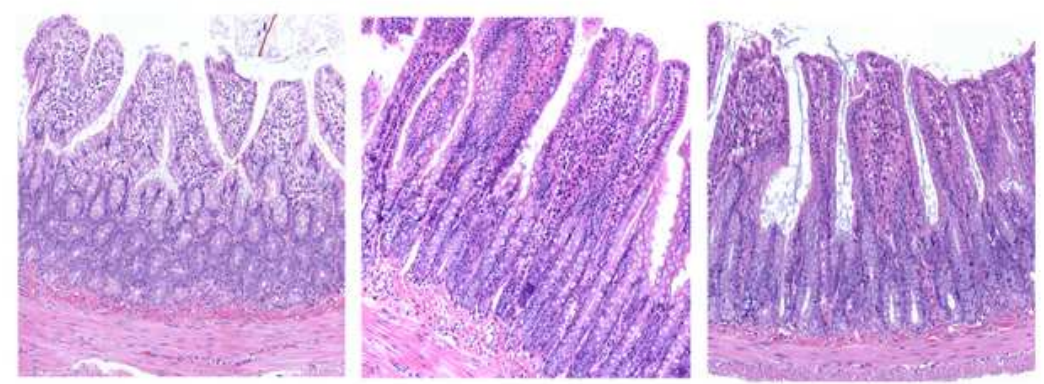

Sham control

IL-37-MSC-treated

MSC-treated

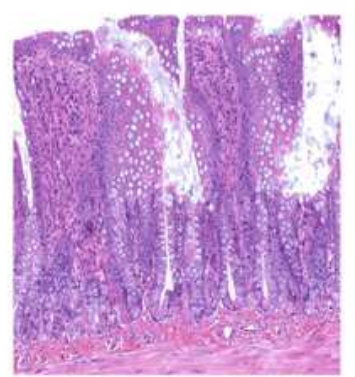

rIL-37-treated

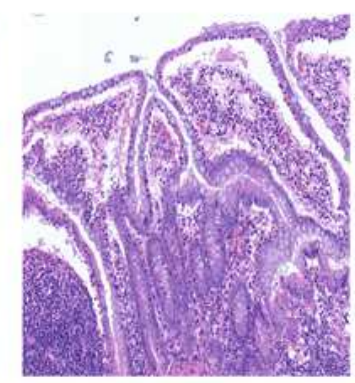

Untreated

b

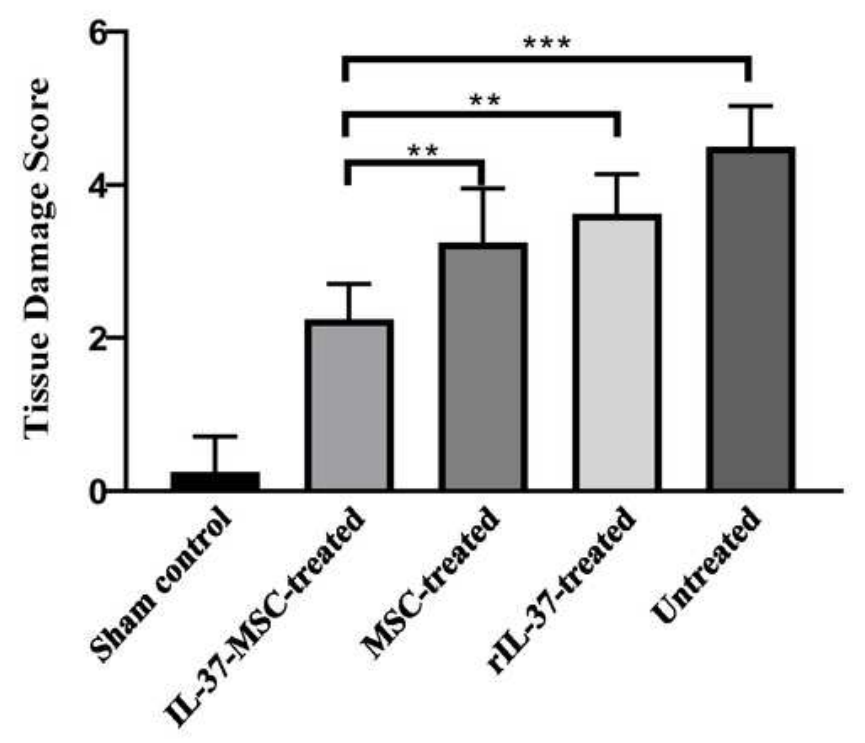

Figure 2

Figure 2 
IL 37 MSCs significantly ameliorated pathological intestine damage following IRI. IL 37 MSCs could Microscopic findings illustrated the architecture of ileum by 72 hours after reperfusion, the damage score was assessed according to Chiu's Score. As shown in Figure 2 a, compared with sham group, untreated group demonstrated severe damage such as inflammatory cells infiltration, hemorrhage and ulcer.

However, as shown in Figure 2 b IL 37 MSC treated IRI group showed more significant therapeutic effects compared with MSC treated and rIL 37 treated groups. The data suggested IL 37 MSCs provide a better protective role in intestinal IRI. Data shown were representative and the $p$ value was determined by one way ANOVA. ${ }^{*} p<0.05, * \star p<0.01, * \star \star p<$ 


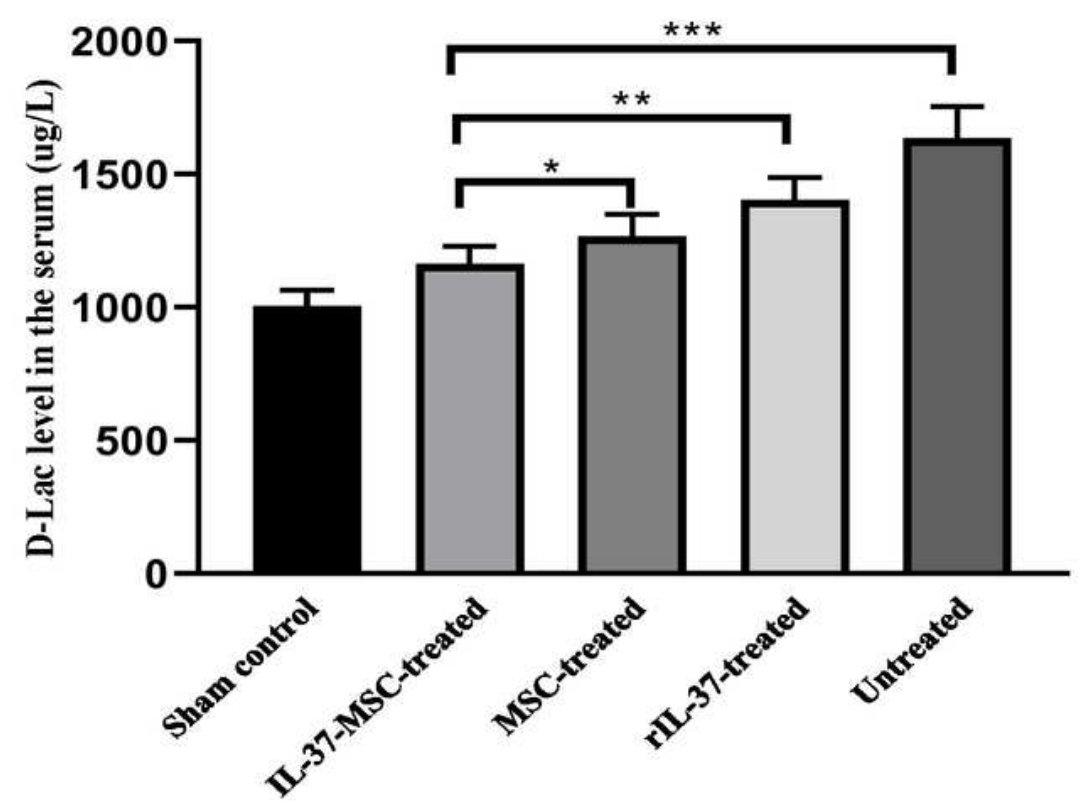

b

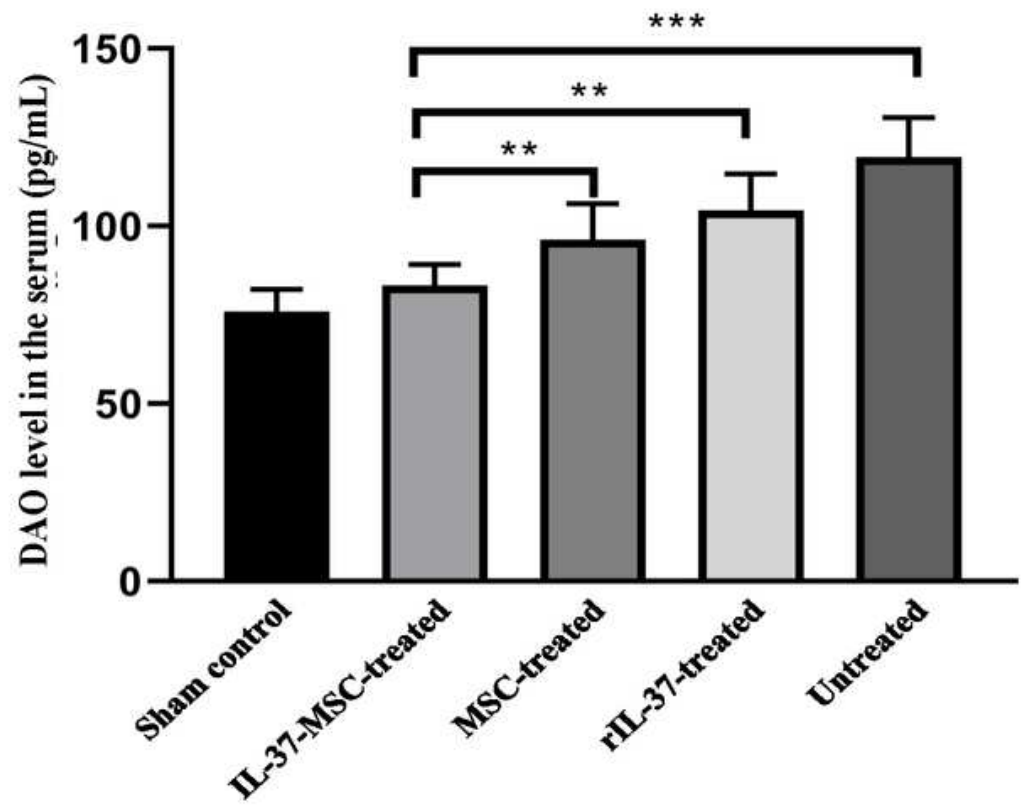

Figure 3

Figure 3

IL 37 MSCs could effectively improve intestinal barrier function following IRI. DAO and D Lac were used to assess the gut barrier function. Serum samples were collected from sham, IL 37 MSC treated, MSC treated, rlL 37 treated and untreated IRI group s. In comparison with MSC treated and rIL 37 treated IRI groups, IL 37 MSCs significantly reduced serum levels of DAO and D Lac, which meant IL 37 MSCs 
remarkedly improved gut barrier function. Data shown were representative and the $p$ value was determined by one way ANOVA. ${ }^{*} p<0.05,{ }^{*} p<0.01, * \star * p<$

a

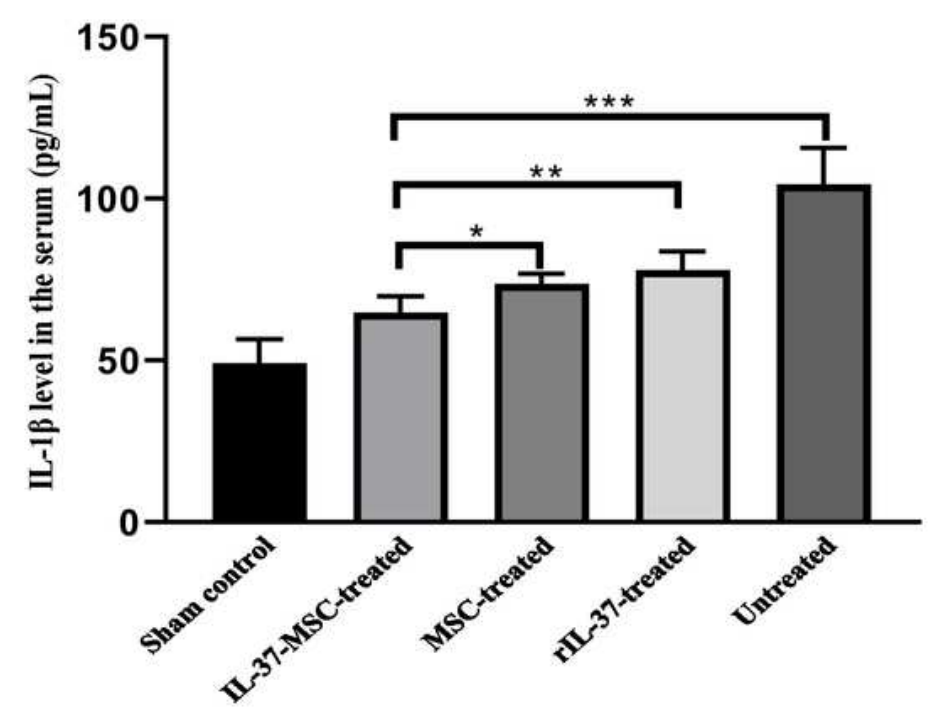

b

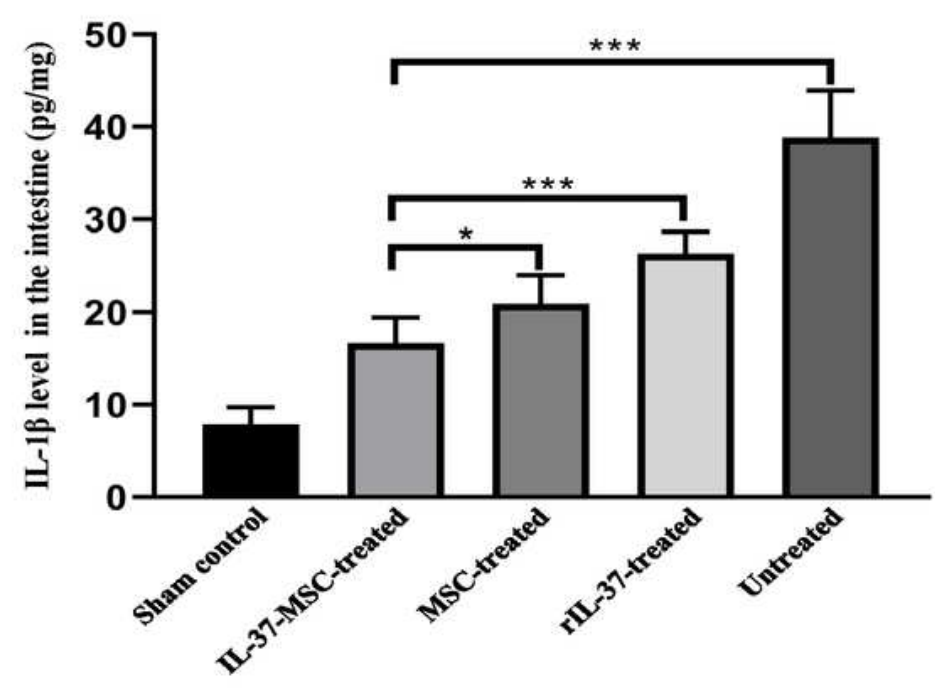

Figure 4

Figure 4

IL 37 MSCs could significantly decrease local and systemic inflammation activity. Local and systemic inflammation activity was assessed by IL $1 \beta$ level in the local tissue and serum. Compared with MSCs 
and rlL 37 treatment, IL 37 MSCs significantly alleviate the local and system inflammation activity. The $p$ value was determined by one way ANOVA. * $p<0.05$, ** $p<0.01$, *** $p<0.001$.

a
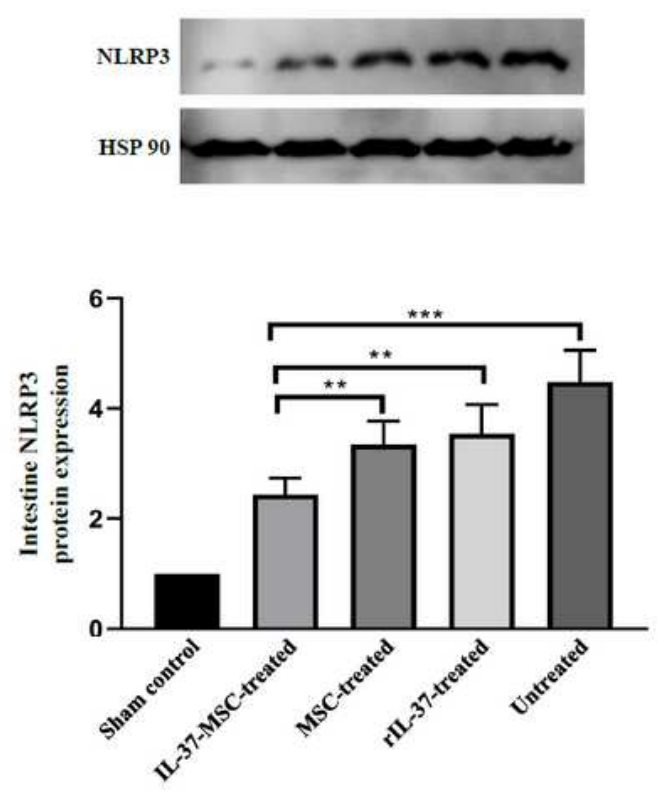

c
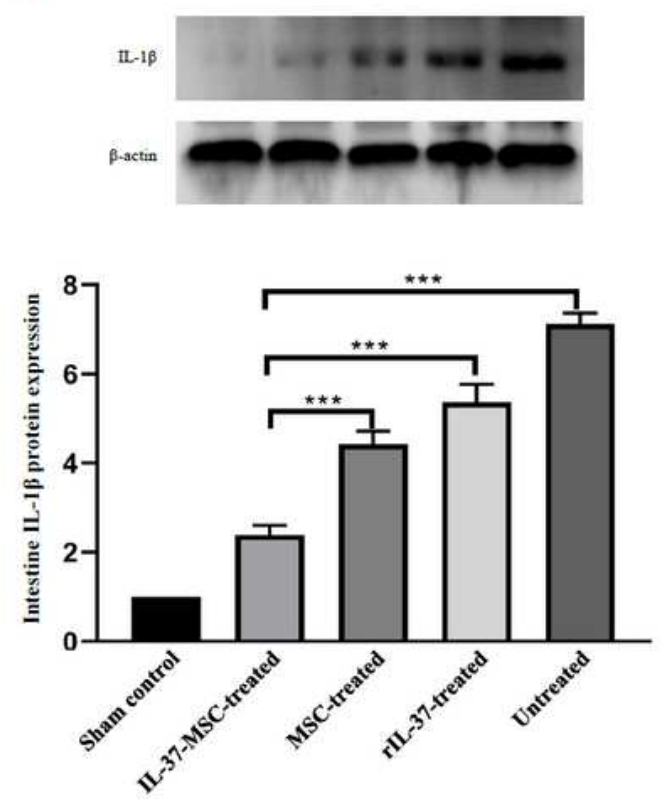

D
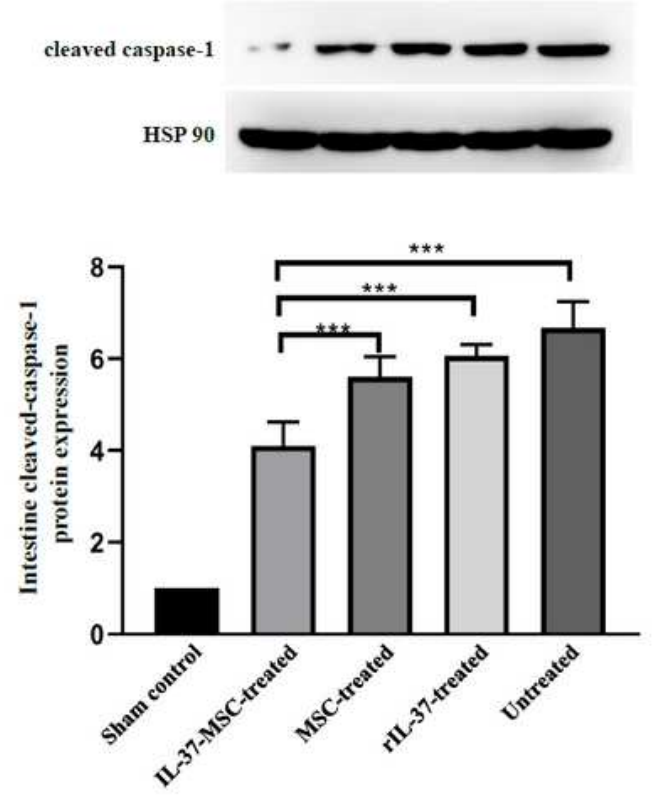

d

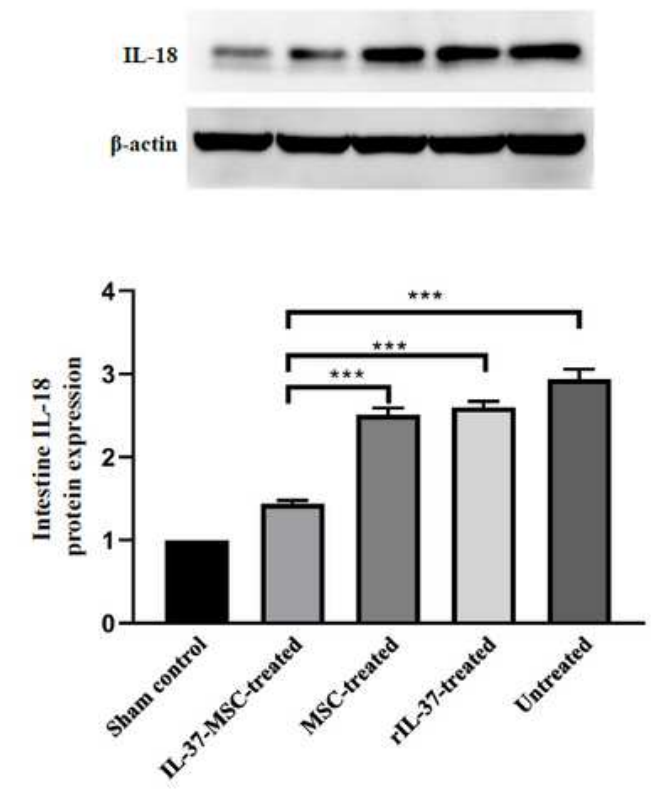

Figure 5

Figure 5

IL 37 MSCs treatment decreased NLRP3 and relative proteins expressions. As shown in Figure $5 \mathrm{a}$, compared with MSCs and IL 37 treatment, IL 37 MSCs significantly decrease $d$ the NLRP3 activation. In paralle I with NLRP3 expression, cleaved caspase 1, IL $1 \beta$ and I L 18 proteins expressions were 
significantly decreased following IL 37 MSCs treatment compared with MSCs and/or rlL 37 treatments (Figure 5b,c \& d), which suggested that IL 37 MSCs could inhibit NLRP3 mediated signaling pathway. Data shown were representative above and the p30 value was determined by one value was determined by one--way ANOVA. * way ANOVA. * $\mathrm{pp}<0.05,{ }^{* \star}<0.05,{ }^{*} \mathrm{pp}<0.01, * \star *<0.01, * \star * \mathrm{pp}<0.001 .<0.001$.

a

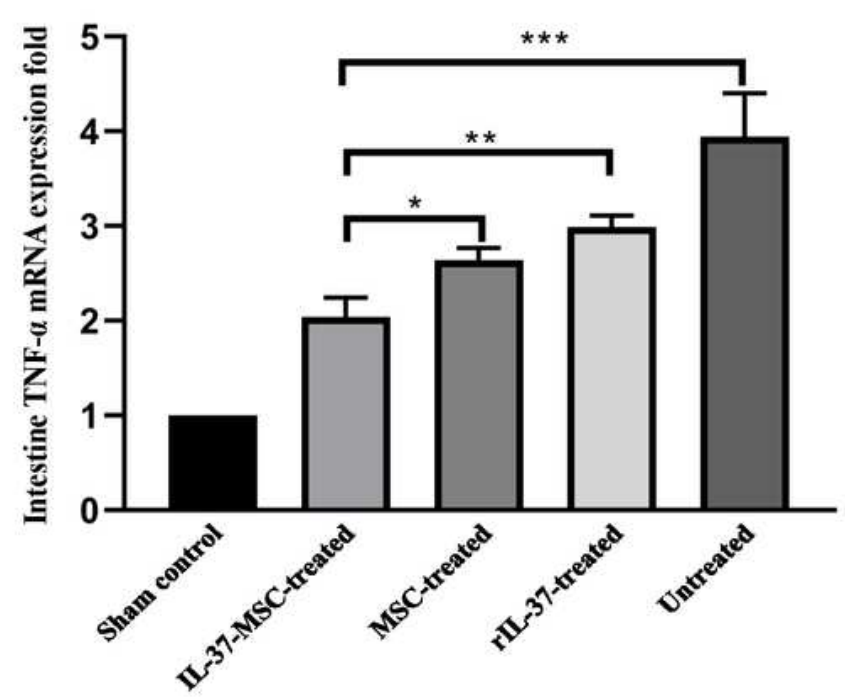

b

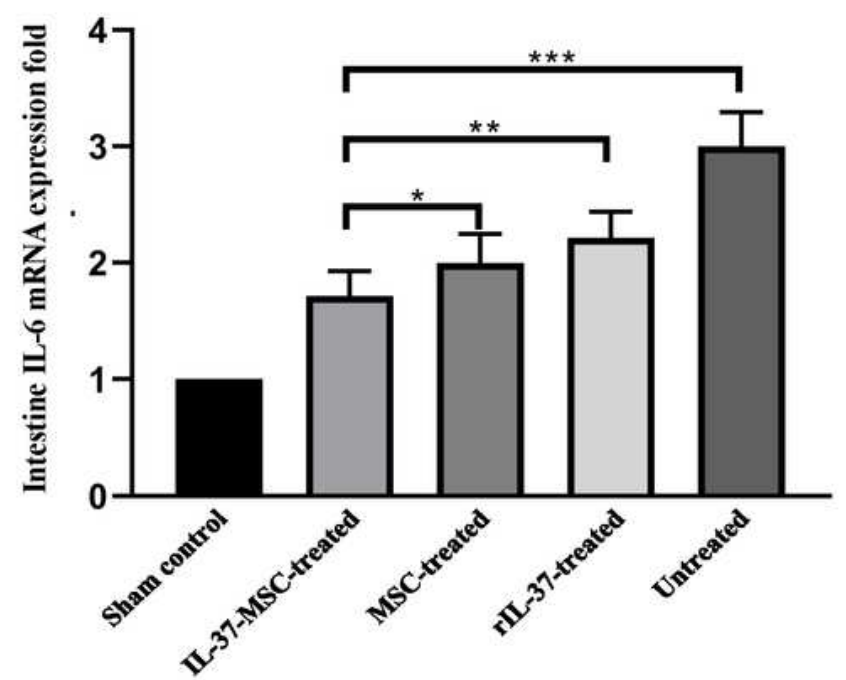

Figure 6

Figure 6 
IL 37 MSCs transplantation decreased IL 6 and TNF a mRNA expression. IL $1 \beta$ and IL 18 are key proinflammatory cytokines, and as their downstream pro inflammation molecules, IL 6 and TNF a play a pivotal role in inflammatory reactivity. As shown in Figure 6 , intestine IL 6 and TNF a mRNA e xpressions in IL 37 MSC treated group w ere significantly decreased compared with MSCs and rlL 37 treated group. The $p$ value was determined by one way ANOVA. * $p<0.05$, $* * p<0.01$, $* \star * p<0.001$.

\section{Supplementary Files}

This is a list of supplementary files associated with this preprint. Click to download.

- Sequence.seq

- SupplementFigure.tif

- SoftwareChromas.rar

- BaseSequence.doc

- Sequence.ab1 